\title{
Environmental impacts of large-scale solar plants on desert region: modeling ground temperature
}

\author{
$\mathrm{Ke} \mathrm{Xu}$, Hengxu Zhang* \\ Key Lab. of Power System Intelligent Dispatch and Control of Ministry of Education (Shandong University), Jinan 250061, China
}

\begin{abstract}
Large-scale PV plants changing the amount of solar radiation received by ground, reducing moisture evaporation, will have influence on ecological environment. This paper used meteorological data from NASA, selected ground temperature as the main object to figure out analytical solution of temperature field and laid a solid foundation of researching relationship between solar plant coverage and environment changing. Firstly, traditional heat transfer formulas are applied to establish the model of the ground temperature. Secondly, the model results are calculated. Finally, data from Golmud is chosen to analyze environmental impacts of large-scale solar plants on arid region. The results show a typical PV plants with a coverage ratio of $60 \%$, ground temperature declines $15^{\circ} \mathrm{C}$ in summer and $6^{\circ} \mathrm{C}$ in winter. The average ground temperature decline in whole year is approximately 3 to $5^{\circ} \mathrm{C}$. It turned out that largescale PV panels will change average ground temperature, which will reduce the air temperature, conserve water and accelerate the growth of plants, thus have a potential influence on environment.
\end{abstract}

Keywords: Solar energy, desert, solar radiation, ground temperature, thermal balance

\section{Introduction}

Energy, environment and fresh water are key factors restricting the sustainable development of our country. Fossil fuel has always been the most important source of energy for hundreds of years. Relying on fossil fuels excessively not only cause serious environmental pollution but also lead challenges of energy depletion. Along with the development of renewable energy power generation technology, largescale solar power is feasible and become backbone of sustained development of society. Solar energy has been the speediest energy technology all over the world especially in developed countries since 2002[1]. Owing to the increasing popularity of solar electricity, costs have already come down by about a factor of four over the past decade [2]. With the development of physics, chemistry, materials science, the efficiency of solar power generation is improving [3]. Solar energy is abundant in China especially in western desert regions. It's appropriate to build large-scale solar energy plant in the region of abundant sunlight and few people.

Large-scale solar power will influence the environment. Researchers domestic and overseas focus on environmental problems during photovoltaic materials production process such as emission of greenhouse gas, a waste of mineral resources and light pollution [4]-[6].

Otherwise, large-scale solar power generation will reduce solar radiation received by ground, decrease ground temperature and lessen soil moisture evaporation, so as to improve the environment. Solar radiation which should travel to the ground is transformed into electricity and transmitted to load center faraway. Environment changes need further study. We select meteorological data from NASA and choose ground temperature as the main object to figure out analytical solution of temperature field, model and analyze environmental impacts of large-scale solar plants on arid regions and laid a foundation of

\footnotetext{
* Manuscript received June 14, 2014; revised July 18, 2014.

Corresponding author: Hengxu Zhang; Tel.: +86-531-88392838; E-mail address: zhanghx@ sdu.edu.cn.

doi: $10.12720 /$ sgce.3.4.390-394
} 
researching relationship between solar plant coverage and environment changing.

The notations used in this paper are listed as Table 1.

Table 1: Notations used in this paper

\begin{tabular}{|c|c|c|c|}
\hline Notation & Definition & Notation & Definition \\
\hline$z$ & Depth inside ground & $B$ & Convective heat transfer coefficient \\
\hline$t$ & Time, $\mathrm{t}=0$ at $6: 00 \mathrm{AM}$ & $\lambda$ & Thermal conductivity \\
\hline$t_{0}$ & $\begin{array}{l}\text { Time difference between maximum solar radiation } \\
\text { time and maximum air temperature time minus } 1 \text {, } \\
\text { generally take } 3\end{array}$ & $Q_{0}$ & $\begin{array}{l}\text { Maximum daily solar radiation intensity, } \\
\text { generally take } Q_{0}=1.31 n Q_{d}\end{array}$ \\
\hline$T_{G}(z, t)$ & Temperature function of ground & $Q_{d}$ & Total amount of daily solar radiation \\
\hline$T_{a}(t)$ & Air temperature function & $a$ & Thermal diffusivity \\
\hline$\overline{T_{a}}$ & Average of daily air temperature & $n$ & Sunshine duration factor \\
\hline$T_{a}$ & Daily Air temperature amplitude & $\omega$ & Time angular frequency, $\omega=2 \pi / 24$ \\
\hline$\alpha_{G}$ & Heat radiation absorption ratio of ground & $u$ & Sunshine duration \\
\hline
\end{tabular}

\section{Modeling Ground Temperature}

The main purpose is to discover the relationships between solar plant coverage and ground temperature. Assume the ground is infinite and its temperature is only determined by depth with the same solar radiation. Temperatures of same depth are the same and direct solar radiation is absorbed by solar panel.

Conduction, convection and radiation are three main forms of heat exchange. In the proposed model of heat transferring, conduction mainly occurs in the solid medium below the surface. Convection mainly refers to the heat flow between ground and air. Radiation is caused by solar radiation and the radiation between air and ground. Generally, the ground absorbs solar radiation in daytime which leads to rise of the temperature. It releases heat at night which causes decrease of ground temperature. Temperature deep inside the ground remains stable as the result of the limitation of heat transferring. Thus, the regional land ground temperature model proposed is based on heat conduction equation and the boundary conditions of heat transferring.

The ground is modeled as an unlimited continuous, homogeneous and isotropic medium. According to laws of heat transfer, the function of heat conduction [7] can be expressed as equation (1). Considering the low fluctuation of temperature deep inside the ground, the lower boundary condition is provided as follows.

$$
\frac{\partial T_{G}(z, t)}{\partial t}=a \frac{\partial^{2} T_{G}(z, t)}{\partial z^{2}}, 0 \leq z \leq \infty \quad z \rightarrow \infty, T_{G}(t)=\text { Const }<\infty
$$

Heat flux balance, which is the upper boundary condition, is obtained as equation (2).

$$
-\left.\lambda \frac{\partial T_{G}}{\partial z}\right|_{z=0}=Q_{G A}+\alpha_{G} Q_{S}
$$

The left part of equation (2) is the heat flux transferring from the ground into underground. While $Q_{G A}$ equals to the heat convection flux between surface and air, which can be expressed as equation (3). Set $\alpha_{G}$ as 0.9 for Golmud.

$$
Q_{G A}=B\left(T_{A}-T_{G}\right)
$$

Suppose the convection mainly refers to natural convection. Thus the value is directly related to wind speed above the ground surface. Set B as $20 \mathrm{KCal} / \mathrm{m}^{2} \cdot h \cdot k$ for the condition in Golmud.

Two sine-wave functions were applied to model the variation of daily air temperature[8] as shown in equation (4). 


$$
T_{a}=\overline{T_{a}}+T_{a}\left[0.96 \sin \omega\left(t-t_{0}\right)+0.146 \sin 2 \omega\left(t-t_{0}\right)\right]
$$

Heat radiation involves the received solar radiation and heat radiation between surface and the air. Due to the weakness of latter radiation form and slight differences between the temperatures of two media, the model proposed applies a revised heat transfer coefficient instead of convection coefficient. The radiation can be equivalently considered to thermal convection. Daily radiation function can be fitted through these data by Fourier series [8], which is shown in equation (5) and (6).For the condition that $n=1$,

$$
Q(\mathrm{t})=\frac{Q_{0}}{\pi}+\frac{Q_{0}}{2} \sin \omega t+\frac{2 Q_{0}}{\pi} \sum_{k=1}^{\infty} \frac{1}{1-4 k^{2}} \sin \left(2 \mathrm{k} \omega \mathrm{t}+\frac{\pi}{2}\right)
$$

For the condition that $n \neq 1$,

$$
Q(\mathrm{t})=\frac{Q_{0}}{n \pi}+\frac{2 n Q_{0}}{\pi} \sum_{k=1}^{\infty} \frac{\cos (\pi \mathrm{k} / 2 n)}{n^{2}-k^{2}} \sin \left(\mathrm{k} \omega \mathrm{t}+\frac{\pi-k \pi}{2}\right)
$$

Temperature of air and ground and solar radiation intensity are all functions of time $t$, the heat balance is expressed as equation (7).

$$
-\left.\lambda \frac{\partial T_{G}(z, t)}{\partial z}\right|_{z=0}=B\left[T_{A}(t)-\left.T_{G}(z, t)\right|_{z=0}\right]+\alpha_{G} Q_{S}(t)
$$

Based on the principle of superposition, the right part of equation (7) can be expressed in the form of Fourier series to solve the temperature function of ground.

\section{Model Implementation and Analysis with PV Plants for Golmud, China}

Climate in Golmud area is the continental plateau climate with dry, windy, and arid. Most importantly, Colmud has a long illumination time with annual average up to 3358 hours, which is extremely suitable for the construction of PV plants. We chose Golmud and the nearby area to construct PV plants. The temperature model is applied to analyze the variation of ground temperature with the influence of PV plants. All the data are obtained from NASA by RETScreen and typical data of four seasons are shown in Table 2.

Table 2. Climate data statistics of Golmud

\begin{tabular}{lllll}
\hline \hline \multicolumn{1}{c}{$\begin{array}{c}\text { Data Name } \\
\text { (Unit) }\end{array}$} & $\begin{array}{c}\text { Typical Day 1 } \\
\text { (Winter) }\end{array}$ & $\begin{array}{c}\text { Typical Day 2 } \\
(\text { Winter) }\end{array}$ & $\begin{array}{c}\text { Typical Day 3 } \\
\text { (Spring) }\end{array}$ & $\begin{array}{c}\text { Typical Day 4 } \\
(\text { Autumn) }\end{array}$ \\
\hline Average air Temperature $\left({ }^{\circ} \mathrm{C}\right)$ & 31.7 & -7.8 & 11.1 & 10.3 \\
Maximum daily air Temperature $\left({ }^{\circ} \mathrm{C}\right)$ & 39.1 & -2.4 & 21.3 & 18.2 \\
Minimum daily air Temperature $\left({ }^{\circ} \mathrm{C}\right)$ & 25 & -11.5 & 2.2 & 5.5 \\
Sunshine duration $(\mathrm{h})$ & 12.5 & 8.6 & 11.3 & 9.5 \\
Total solar radiation in horizon $\left(\mathrm{MJ} /\left(\mathrm{m}^{2} \cdot 24 \mathrm{~h}\right)\right)$ & 29.99 & 9.07 & 25.74 & 14.83 \\
\hline \hline
\end{tabular}

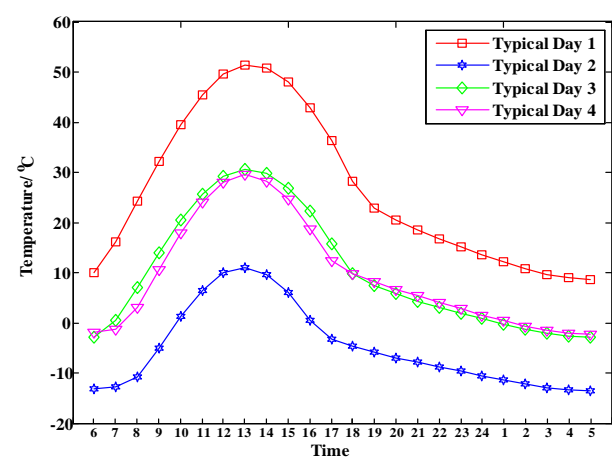

(a)

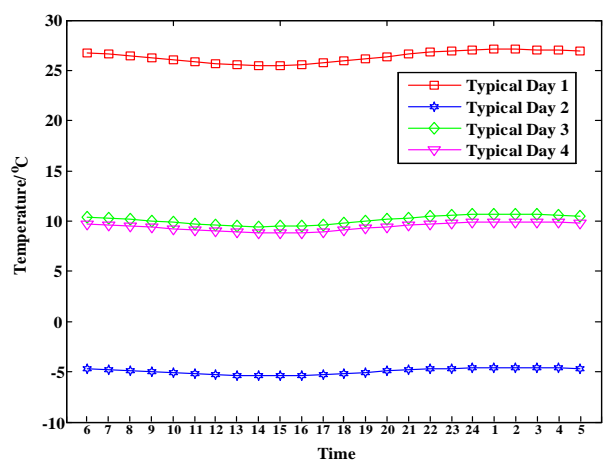

(b)

Fig. 1. Daily temperature curve of ground at: (a) $z=0 \mathrm{~cm}$ and (b) $z=40 \mathrm{~cm}$. 


\subsection{Original ground temperature}

The original ground temperature can be calculated through simulation. The curves are plotted in Fig. 1 at $z=0$ and $40 \mathrm{~cm}$. The temperature varies more significantly in summer than other seasons at $z=0 \mathrm{~cm}$, while temperature is stable in deep underground.

Taking typical day 1 to do more analysis, different temperature curves with different depths are plotted in Fig. 2. It indicates that temperature variation is about 3 to 4 hours ahead as the depth increases $10 \mathrm{~cm}$. Temperature at $z=40 \mathrm{~cm}$ is stable.

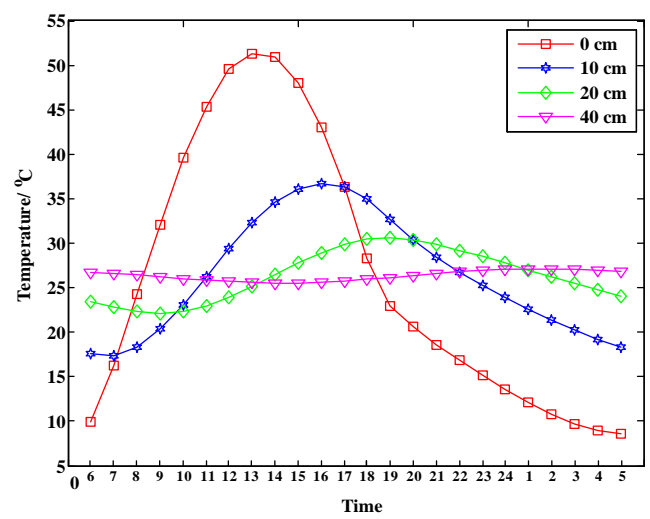

Fig. 2. Temperature curves vary with depths.

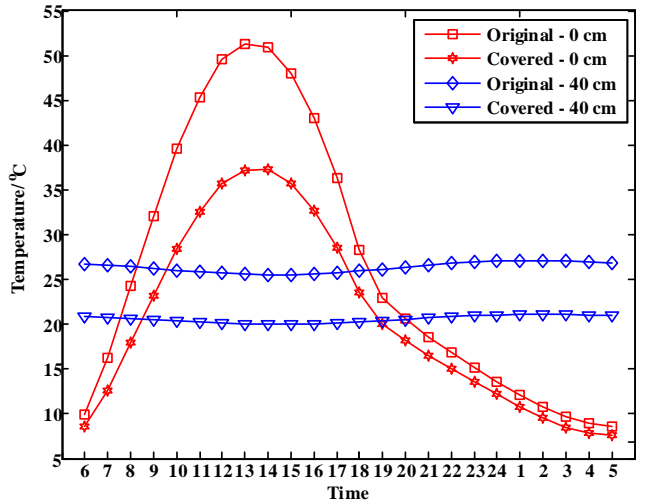

Fig. 3. Daily temperature curves comparison.

\section{Ground Temperature Analysis Covered by PV Panels}

Generally, solar radiation includes direct and scattered components. After the construction of PV plants, PV panels cover huge region and absorb solar radiation, especially the direct component. As a result, only part of direct components and all the scattered components of radiation is received by ground. Suppose the ratio of scattered components is $40 \%$ in Golmud, and the ratio of covered land is $60 \%$. Thus, the ratio of received radiation by ground is approximately $64 \%$. The relating temperature curves are plotted in Fig. 3, which shows that the temperature of ground declines in varying degrees at different depths. Temperature in covered area is $15^{\circ} \mathrm{C}$ lower than the bare areas at $z=0 \mathrm{~cm}$ and $6^{\circ} \mathrm{C}$ lower at $z=40$ $\mathrm{cm}$. Considering different density of PV panels, different temperature curves with different coverage ratio are plotted in Fig. 4. It can be concluded that the ground temperature drops with the increase of coverage ratio. With the whole year data obtained, the average temperature decline of ground is shown in Fig. 5. A general influence of PV plants to the temperature of ground can be obtained that the average decline is about $3 \sim 5^{\circ} \mathrm{C}$. The decline is more significant in summer than that in winter.

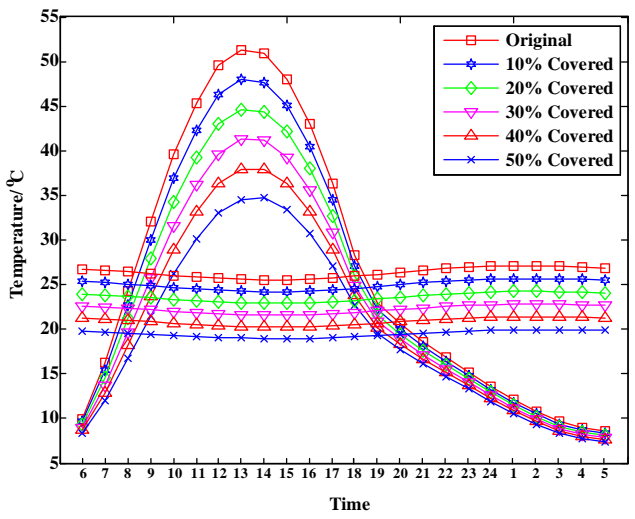

Fig. 4. Temperature curves with different coverage ratio.

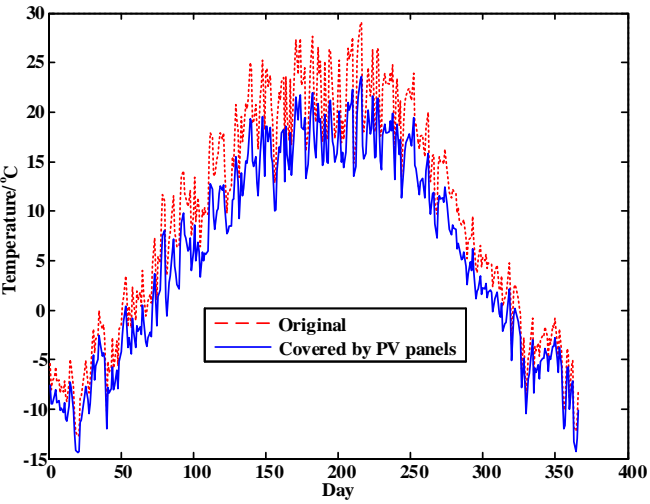

Fig. 5. Average temperature decline of ground 


\section{Conclusions}

This paper proposes a temperature model of ground with the consideration of PV panel coverage. The model is also applied to analyze the temperature decline of ground with NASA's data. The results indicate that large-scale PV plants will lower the temperature of ground significantly in local region. The case proposed shows a typical PV plants with a coverage ratio of $60 \%$, temperature declines $15^{\circ} \mathrm{C}$ in summer and $6^{\circ} \mathrm{C}$ in winter. The average temperature decline in whole year is approximately 3 to $5^{\circ} \mathrm{C}$.

Large-scale PV plants in deserts with much solar radiation, less precipitation and evaporation can utilize the solar energy and lower the temperature of ground. It may influence evaporation, air temperature and the vegetation in other aspects. Paper [9] indicates vegetation index, ground temperature are significantly interacted in arid and semi-arid regions.

However, results are only in view of the model in this paper, the emphasis is to build a more accurate model in the future. PV panel coverage is a method of human intervention to natural environment. Given the facts with fragile ecological environment of desert region, further research of the impacts on surface characteristics and ecological environment with large-scale PV plants construction in desert regions is on schedule.

\section{Acknowledgements}

This work is supported by the National High Technology Research and Development Program of China (863 Program, No.2011AA05A118).

\section{References}

[1] Devabhaktuni V, Alam M, Shekara Sreenadh Reddy Depuru S, et al. Solar energy: Trends and enabling technologies. Renewable and Sustainable Energy Reviews, 2013; 19:555-564.

[2] Solar energy's path towards competitiveness. Nat Mater, 2012; 11:173-173.

[3] Heffernan O. A bright future for solar power. Nature Reports Climate Change. 2008.

[4] Alsema E A, de Wild-Scholten M J, Fthenakis V M. Environmental impacts of PV electricity generation-a critical comparison of energy supply options[C]. In: Proc. 21 st European Photovoltaic Solar Energy Conference, Dresden, Germany; 2006.

[5] Tsoutsos T, Frantzeskaki N, Gekas V. Environmental impacts from the solar energy technologies. Energy Policy, 2005; 33:289-296

[6] Turney D, Fthenakis V. Environmental impacts from the installation and operation of large-scale solar power plants. Renew Sust Energ Rev, 2011; 15:3261-3270.

[7] Holman J P. Heat Transfer. Mc Gran-Hill Book Company, Soythern Methodist University; 1986.

[8] Yan Zuoren. Analysis of the temperature field in layered pavement system. Journal of Tongji University, 1984:76-85.

[9] Study on NDVI-T_s space by combining LAI and evapotranspiration. Science in China, (Series D:Earth Sciences), 2006:747754. 